\title{
What drives inhaler prescription for asthma patients? Results from a real-life retrospective analysis
}

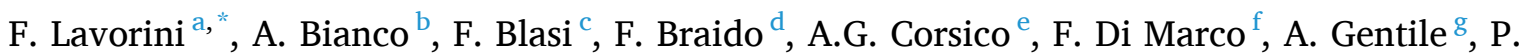 \\ L. Paggiaro $^{\text {h }}$, V. Pegoraro ${ }^{i}$, G. Pelaia ${ }^{j}$, P. Rogliani ${ }^{k}$, P. Santus ${ }^{1}$, N. Scichilone ${ }^{\mathrm{m}}$, A. Soldi ${ }^{g}$, G. \\ W. Canonica ${ }^{\mathrm{n}}$
}

a Department of Experimental and Clinical Medicine, University of Florence, Florence, Italy

b Department of Translational Medical Sciences, University of Campania "L. Vanvitelli", Naples, Italy

${ }^{\mathrm{c}}$ Fondazione IRCCS Cà Granda Ospedale Maggiore Policlinico Internal Medicine Department, Respiratory Unit and Adult Cystic Fibrosis Center, Department of

Pathophysiology and Transplantation, Università degli Studi di Milano, Milan, Italy

${ }^{\mathrm{d}}$ Department of Internal Medicine, Respiratory Diseases and Allergy Clinic, University of Genova, Azienda Policlinico IRCCs San Martino, Genoa, Italy

${ }^{\mathrm{e}}$ Division of Respiratory Diseases, IRCCS Policlinico San Matteo Foundation - Department of Internal Medicine and Therapeutics, University of Pavia, Pavia, Italy

${ }^{\mathrm{f}}$ Department of Health Sciences, University of Milan, Italy - Respiratory Unit, Papa Giovanni XXIII Hospital, Bergamo, Italy

${ }^{\mathrm{g}}$ Mundipharma Pharmaceuticals, Milan, Italy

${ }^{\mathrm{h}}$ Department of Surgery, Medicine, Molecular Biology and Critical Care, University of Pisa, Pisa, Italy

i IQVIA Solutions Italy, Milan, Italy

j Department of Medical and Surgical Sciences, University "Magna Graecia” of Catanzaro, Catanzaro, Italy

${ }^{\mathrm{k}}$ Respiratory Medicine Department of Experimental Medicine, University of Rome "Tor Vergata" - Respiratory Unit, Policlinico Tor Vergata, Rome, Italy

${ }^{1}$ Department of Biomedical and Clinical Sciences (DIBIC) - University of Milan. Division of Respiratory Diseases - "L. Sacco" University Hospital - ASST Fatebenefratelli Sacco - Milan, Italy

m PROMISE Department, University of Palermo, Palermo, Italy

${ }^{\mathrm{n}}$ Personalized Medicine, Asthma and Allergy Clinic, Humanitas Research Hospital - Department of Biomedical Sciences, Humanitas University, Milan, Italy

\section{A R T I C L E I N F O}

\section{Keywords:}

Inhalers

Asthma

Pressurised metered-dose inhalers

dry powder inhalers

General practitioners

Respiratory specialists

\begin{abstract}
A B S T R A C T
Background: The choice of inhaler device for asthma patients depends upon multiple attributes. We investigated factors that may drive general practitioners (GPs) and respiratory specialists in the prescription of inhaler devices for asthma patients who initiated inhalation therapy.

Methods: We retrospectively analysed prescriptions by GPs and respiratory specialists to asthma patients commencing inhaled corticosteroid/long-acting $\beta 2$-agonist combination therapy available as both pressurised metered-dose inhalers (pMDIs) and dry powder inhalers (DPIs). Patient characteristics were compared by device and multivariate analysis was used to model the likelihood of receiving a pMDI as opposed to a DPI in order to identify drivers for prescription. A sample of the respiratory specialists completed an ad-hoc survey of their perceived success in achieving asthma control in their patients and barriers to attaining full control.

Results: Prescription of a particular inhaler device was unrelated to the characteristics of the patients. Multivariate analysis revealed that the main driver for the choice of inhaler device choice was the medication (Odds Ratio and 95\% Confidence Interval, respectively for GPs and specialists: 0.19 [0.16-0.23]; 0.17 [0.08-0.37]). Specialists perceived asthma as being inadequately controlled in $41 \%$ of their patients, and considered patients' difficulties in using DPIs and pMDIs as instrumental in this, citing a need for a novel, more effective inhaler technology.

Conclusion: Physicians choose inhaler devices according to the prescribed drugs and not to the characteristics of the individual patient. This may reflect a lack of confidence in existing inhaler devices and underlines the need for technologies, which are more reliable and easier to use by patients.
\end{abstract}

\footnotetext{
* Corresponding author.

E-mail address: federico.lavorini@unifi.it (F. Lavorini).
} 


\section{Introduction}

Current asthma guidelines recommend inhaled corticosteroid and long-acting $\beta 2$-agonist (ICS/LABA) fixed-dose medications as controller medication for the management of patients with moderate (i.e. step 3 and 4) disease [1]. Although ICS/LABA medications have been shown to relieve asthma symptoms [2,3], they need to be delivered regularly and in sufficient quantities to the airways in order to be effective. Suboptimal adherence to inhaled medications continues to be a significant barrier to asthma control, contributing to the deterioration of symptoms and quality of life as well as costly exacerbations over time [4,5]. Good adherence in turn requires patients' acceptance of their need for regular and continuous inhaled therapy, together with the successful mastery of the inhaler technique specific to their device(s) [5]. Accumulating evidence now suggests that the quality of inhaler technique itself influences adherence: indeed one might anticipate that patients are less likely to adhere to a treatment that they find apparently ineffective because they cannot or do not use it properly [6-9]. Thus, devices used for inhalation therapy in asthma may critically affect outcomes, through both poor drug delivery and poor adherence, independently of the actual medication used [5]. The effectiveness of any individual device in delivering the contained drug(s) to the airways may depend on several factors, mainly its ability to deliver, by inhalation, a sufficient respirable fraction of the delivered drug(s) to the airways reproducibly, precisely and stably over time $[9,10]$. Ease of use may also be critical, particularly in patients at the extremes of age, those with physical disabilities and those with limited inspiratory capacity $[9,10]$. The wide variety of inhaler devices now available presents both opportunities and challenges for patients and prescribers. Indeed, in addition to the broad and fundamental differences in the correct usage of pressurised, metered-dose inhalers (pMDIs) and dry powder inhalers (DPIs), every single device within these categories may require specific nuances to optimize continued, optimal and sufficient drug delivery $[9,10]$. Both types of device are available to deliver ICS/LABA combination therapy: pMDIs are widely prescribed because of their low cost, and use consistent technology to deliver a variety of medications relatively independently of inhalational effort $[9,10]$. However, they require coordination of inhaler actuation and inhalation, and although this can often be accomplished relatively easily with a spacer device, there is room for error even after repeated, appropriate training [11]. In contrast, DPIs do not require coordination of inhaler actuation with inhalation since they are actuated by the patient's inspiratory flow $[9,10,12]$. The very fact that DPIs are inspiratory flow driven does however necessitate a forceful and deep inhalation to de-agglomerate the powder formulation into respirable particles to ensure that drug is delivered to the lungs [12]. Therefore, DPIs may not be effective for the treatment of patients with severe airflow limitation [13].

Despite studies highlighting the importance when treating asthma by tailoring devices to the individual characteristics of each patient [14-16], in the "real world" the choice of device appears to be governed principally by the type of medication used $[17,18]$. In practice, the choice of a therapeutic agent and the device used to deliver it lie with the prescribing physician and should strike a balance between appropriate medication and selection of the inhalation device [19]. It has to be taken into consideration that the choice of the most appropriate inhaler device for each patient has been claimed to be as critical as the choice of medication itself [15].Thus, the prescriber should be able to tailor the selection of the device to the individual patient, evaluating needs, functional ability, and the complexity of the medication regimen [19].

Given the key role of inhaler devices and healthcare providers in the management of asthma, this study aimed to explore factors influencing the choice of inhaler device by general practitioners (GPs) and specialists when initiating therapy for asthma, using real-world data. We then surveyed a sample of specialists to understand their perception of currently available inhaler devices and novel inhaler technologies. A greater understanding of the relationship between patient characteristics and inhaler choice may facilitate efforts to identify patients at risk of incorrect inhaler usage, and in turn guide clinicians in prescribing, for each individual patient, a device that is most likely to be used correctly.

\section{Methods}

\subsection{Study design and data collection}

We retrospectively investigated the characteristics of asthma patients prescribed different inhaler devices by interrogating the IQVIA Longitudinal Patient Database (LPD) and the IQVIA Patient Analyzer Database (PAD). IQVIA (www.iqvia.com) is an international healthcare information company specialising in the collection and interpretation of anonymous health information, and often the only source of information on aspects of medicinal use across the world. The IQVIA LPD is a computerized network of GPs from different European countries providing information to a centralised database on patients' consultations and treatments. This database reflects the clinical practice of a national sample of GPs since it allows the collection and longitudinal analysis of data taken from patients' records related to prescription practices and healthcare utilisation in everyday clinical practice. Drug prescriptions and medical diagnoses are both coded directly by GPs. Drug prescriptions comply with the Anatomical Therapeutic and Chemical (ATC) classification system, while medical diagnoses comply with the 9th edition of International Classification of Disease (ICD-9CM). Currently, about 900 Italian GPs contribute to the IQVIA LPD, providing data from routinely collected records of $\sim 1.2$ million patients. The Italian IQVIA LPD has been shown to be a reliable source of information in documented in a range of studies of different diseases [20-25], including respiratory diseases [26,27]. From the IQVIA LPD we retrieved data on patients coded in 2016 (the selection period) with a diagnosis of asthma (ICD-9-CM 493.xx) and at least one prescription of beclomethasone dipropionate (BDP)/formoterol or fluticasone/salmeterol fixed dose combination (study cohort) delivered by either a DPI or by a pMDI. The study was restricted to these ICS/LABA combinations because, at the time of the study, they were the only ICL/LABA combinations available as both pMDIs and DPIs. Patients who were prescribed both DPI and pMDI inhaler devices were excluded. For each patient, the date of the first $\mathrm{BDP} /$ formoterol or fluticasone/salmeterol prescription during the selection period was indicated as the Index Date. For each patient of each study group, we collected information on clinical characteristics such as gender, age, body mass index (BMI), smoking history and relevant co-morbidities (i.e. cardiovascular or neurologic diseases, diabetes, dyslipidaemia, osteoporosis, gastro-oesophageal reflux, allergic rhinitis, sinusitis, nasal polyps, personality and non-psychotic mental disorders) during the 12 months preceding the Index Date. Spirometry testing, specialist examinations, prescriptions of oral corticosteroids, asthma-related antibiotics, exacerbation [1], hospitalisations or emergency room admissions for asthma were also collected during the 12 months after the Index Date.

The IQVIA PAD contains data from asthma patients obtained by means of a web questionnaire administered to Italian pulmonologists who collected medical records of patients evaluated during a single week, thereby providing a snapshot of real-life clinical activity [27]. As for the LPD, from the IQVIA PAD we retrieved data from asthma patients initiating in 2016 treatment with a $\mathrm{BDP} /$ formoterol or a fluticasone/salmeterol combination administered by either a DPI or by a pMDI. Specialists were asked for information on patient characteristics, asthma severity and control, as well as spirometric data (i.e. forced expiratory volume in the first second $\left(\mathrm{FEV}_{1}\right)$ and peak expiratory flow (PEF) measurements).

We also planned an ad-hoc survey on a sample of the specialists who had participated in the IQVIA PAD to investigate their perception of the quality of asthma control attained by their patients, the reason(s) why asthma remained uncontrolled and factors influencing their prescription 
of DPIs or pMDIs. The survey, based on a web questionnaire (see online supplement), also evaluated the specialists' knowledge about currently available pMDIs and DPIs, and sought their opinion on the novel breathtriggered MDI K-haler ${ }^{\circledR}$ (Mundipharma International, UK), a new inhaler device that, due to its technology [28], may improve device usability by patients [29]. Additional details on the methods are reported in the online supplement.

\subsection{Statistical analysis}

Analyses using IQVIA LPD and PAD data were stratified by device type (pMDI or DPI). Descriptive statistics were presented and Chi-square tests or T-tests for independent groups were applied to evaluate the association between device type and each collected variable, as appropriate. Multivariate logistic models were applied to evaluate factors that could influence device choice. Device type was set as the dependent variable, while covariates included age, gender, variables significantly associated with device type (following Chi-square or T-test analyses), and variables recommended from scientific literature to take account when choosing the device (when available). Finally, all variables collected through the ad-hoc web questionnaire were descriptively analysed according to the nature of the corresponding questions. In particular, qualitative variables were described using frequencies and percentages, while quantitative variables were described in terms of mean value and standard deviation. All analyses were performed on anonymised data using SAS software (Version 9.4). A p-value of $<0.05$ was considered statistically significant.

\section{Results}

\subsection{Baseline characteristics of patients from the GP and specialist} databases

The number of asthmatic patients meeting inclusion criteria were 4979 for the IQVIA LPD study cohort and 660 for the IQVIA PAD study cohort. Overall, the proportion of patients prescribed a pMDI was higher than those prescribed a DPI in both GP ( $57.6 \%$ versus $42.4 \%)$ and specialist (57.4\% versus $42.6 \%$ ) databases. However, when comparing patients prescribed BDP/formoterol with those prescribed fluticasone/ salmeterol, we found that in both GP and specialist databases, significantly more patients who were prescribed BDP/formoterol received a pMDI, whereas patients who were prescribed fluticasone/salmeterol more frequently received a DPI (Fig. $1, \mathrm{P}$ always $<0.05$ ). Table 1 shows baseline (i.e. before initiating treatment with an ICS/LABA combination) characteristics of patients stratified by the device type prescribed. Overall, patients in the specialist database were younger and had fewer co-morbidities than those in the GP database. For both GPs' and specialists' patients, those receiving a DPI were slightly but significantly (P $<0.05$ ) older than those receiving a pMDI. Patients prescribed DPIs by
GPs had a slight but significantly $(\mathrm{P}<0.05)$ higher BMI than those prescribed pMDIs. A higher proportion of smokers was observed among patients prescribed DPIs by specialists $(29.89 \%$ vs. $9.91 \%, \mathrm{P}<0.05)$, while no differences in terms of comorbidities were observed between patients prescribed a pMDI and those prescribed a DPI in either cohort (Table 1). Finally, focusing on specialists' patients, no differences in terms of $\mathrm{FEV}_{1}$ and PEF values were observed between the DPI and PMDI groups (data not shown).

\subsection{Multivariate regression to examine predictors of inhaler choice}

With regard to GPs' patients, multivariate logistic analysis revealed that gender, age, and comorbidities did not influence the choice of inhaler device (Table 2). Higher BMI values appeared to result in a slight but significant $(\mathrm{P}<0.05)$ increase in the likelihood of receiving a DPI, although this was clinically negligible (i.e. a 1 point increase in BMI corresponded to a $2 \%$ increase in the likelihood of receiving a DPI rather than a pMDI). Patients prescribed $\mathrm{BDP} /$ formoterol had a significantly ( $\mathrm{P}$ $<0.05$ ) lower likelihood of receiving a DPI rather than a pMDI when compared to patients prescribed fluticasone/salmeterol (about $80 \%$ less).

Using the specialists' database, both age and medication influenced the choice of device, while gender, co-morbidities, smoking and PEF had no effect. In particular, the likelihood of receiving a DPI increased according to the age of the patient. However, the wide range in confidence intervals for odds ratio estimates seemed to suggest some variability in the prescribing behaviour of the specialists with regard to patients' age. Again, a strong influence of the medication on device choice was observed, with an effect magnitude and direction similar to that observed for the GPs' patients (Table 2).

\subsection{2-Month follow-up on GPs' patients stratified by type of device}

Table 3 reports information collected during the 12-month follow-up period following the initiation of the LABA/ICS treatment, for the GPs' patients. No differences were observed between the pMDI and DPI groups in terms of resource utilisation (i.e. spirometry and specialist visit requests, hospitalisations) and exacerbations. The only exception was short-acting beta agonist (SABA) co-prescriptions, as the proportion of patients who received them was significantly higher for the DPI group than the pMDI group. Overall, the proportion of patients with spirometry and/or specialist visit requests was quite low (about 15\%), while the proportion of patients experiencing exacerbations was quite high (about 21\%; Table 3).

\subsection{Specialists' web-based ad-hoc survey on pMDIs and DPIs}

Fifty-one specialists across Italy participated in the ad-hoc survey. From medical records, asthma was regarded as not well controlled in

\section{Specialists}

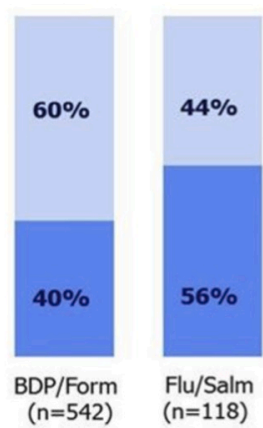

$P<0.05$ for both groups

$(n=542) \quad(n=118)$

Fig. 1. Proportion of patients stratified by ICS/LABA medication and device type prescribed by general practitioners (GPs) and specialists. 
Table 1

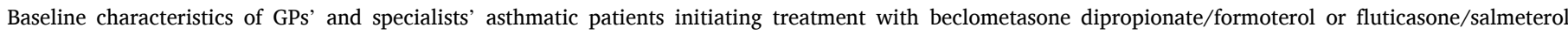
stratified by device type.

\begin{tabular}{|c|c|c|c|c|c|c|c|c|c|c|c|c|c|c|}
\hline & \multicolumn{6}{|c|}{ GPs' Patients } & \multicolumn{8}{|c|}{ Specialists' Patients } \\
\hline & \multicolumn{2}{|c|}{ Total $(\mathrm{N}=4979)$} & \multicolumn{2}{|c|}{ pMDI $(\mathrm{N}=2870)$} & \multicolumn{2}{|c|}{ DPI $(\mathrm{N}=2109)$} & \multicolumn{2}{|c|}{ Total $(\mathrm{N}=660)$} & \multicolumn{2}{|c|}{ pMDI $(\mathrm{N}=379)$} & \multicolumn{3}{|c|}{ DPI $(\mathrm{N}=281)$} & \\
\hline Female: N (\%) & 2914 & $(58.53 \%)$ & 1.653 & $(57.60 \%)$ & 1261 & $(59.79 \%)$ & & 380 & $(57.58)$ & 217 & $(57.26)$ & 163 & (58.01) & \\
\hline \multicolumn{15}{|l|}{ Age } \\
\hline Years: Mean (SD) & 48.30 & (18.30) & 47.30 & (18.20) & 49.60 & (18.40) & $*$ & 36.25 & (16.38) & 35.75 & (17.21) & 36.93 & $(15.20)$ & \\
\hline$\leq 18$ yrs: $\mathrm{N}(\%)$ & 233 & $(4.68 \%)$ & 145 & $(5.05 \%)$ & 88 & $(4.17 \%)$ & & 84 & (12.73) & 61 & (16.09) & 23 & $(8.19)$ & \\
\hline 18 -| 65 yrs: N (\%) & 3766 & $(75.64 \%)$ & 2.204 & $(76.79 \%)$ & 1.562 & $(74.06 \%)$ & $*$ & 542 & (82.12) & 300 & (79.16) & 242 & (86.12) & * \\
\hline$>65$ yrs: $\mathrm{N}(\%)$ & 980 & $(19.68 \%)$ & 521 & $(18.15 \%)$ & 459 & $(21.76 \%)$ & & 34 & $(5.15)$ & 18 & $(4.75)$ & 16 & $(5.69)$ & \\
\hline \multicolumn{15}{|l|}{ Body Mass Index } \\
\hline $\mathrm{Kg} / \mathrm{m}^{2}:$ Mean (SD) & 26.80 & $(5.80)$ & 26.50 & $(5.70)$ & 27.10 & $(6.00)$ & $*$ & 23.91 & (3.65) & 23.79 & (3.51) & 24.07 & (3.84) & \\
\hline Missing: N (\%) & 2222 & $(44.63 \%)$ & 1313 & $(45.75 \%)$ & 909 & $(43.10 \%)$ & & 0 & . & 0 & . & 0 & . & \\
\hline \multicolumn{15}{|l|}{ Smoking Habits } \\
\hline Smokers: N (\%) & 513 & $(10.30 \%)$ & 304 & $(10.59 \%)$ & 209 & $(9.91 \%)$ & & 165 & $(25.00)$ & 81 & (21.37) & 84 & (29.89) & * \\
\hline \multicolumn{15}{|l|}{ Comorbidities $^{1}$} \\
\hline No comorbidities: N (\%) & 2639 & $(53.00 \%)$ & 1548 & $(53.94 \%)$ & 1091 & $(51.73 \%)$ & & 530 & $(80.30)$ & 304 & $(80.21)$ & 226 & $(80.43)$ & \\
\hline 1 co-morbidity: $\mathrm{N}(\%)$ & 1345 & $(27.01 \%)$ & 767 & $(26.72 \%)$ & 578 & $(27.41 \%)$ & & 123 & (18.64) & 72 & (19.00) & 51 & (18.15) & \\
\hline $2+$ co-morbidities: $\mathrm{N}(\%$ & 995 & $(19.98 \%)$ & 555 & $(19.34 \%)$ & 440 & $(20.86 \%)$ & & 7 & (1.06) & 3 & $(0.79)$ & 4 & $(1.42)$ & \\
\hline
\end{tabular}

*p < 0.05 comparing pMDI vs DPI groups. DPI: dry powder inhaler; GP: general practitioner; pMDI: pressurised, metered-dose inhaler; SD: standard deviation.

1 Co-morbidities included ischaemic heart disease, disease of the pulmonary circulation, cardiac arrhythmia, heart failure, systemic hypertension, diabetes mellitus,

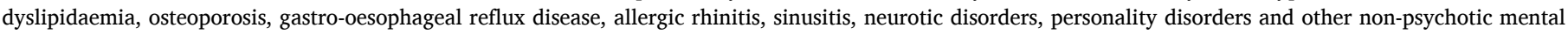
disorders, and nasal polyps.

Table 2

Multivariate logistic models evaluating factors that may influence the choice of the device (DPI versus pMDI) by GPs and specialists.

\begin{tabular}{|c|c|c|}
\hline \multicolumn{2}{|l|}{ GPs $(N=2757)$} & \multirow{2}{*}{$\begin{array}{l}\text { OR }[95 \% \mathrm{CI}] \\
1.00\end{array}$} \\
\hline Gender & Female & \\
\hline & Male & $0.96[0.81-1.13]$ \\
\hline \multirow[t]{3}{*}{ Age } & $<18$ years & 1.00 \\
\hline & 18-65 years & $1.35[0.88-2.07]$ \\
\hline & $>65$ years & $1.40[0.87-2.25]$ \\
\hline \multirow[t]{3}{*}{ Comorbidities } & No comorbidities & 1.00 \\
\hline & 1 co-morbidity & $1.05[0.86-1.28]$ \\
\hline & At least 2 co-morbidities & $0.96[0.76-1.21]$ \\
\hline BMI & & $1.02[1.01-1.04]^{*}$ \\
\hline \multirow[t]{2}{*}{ Medication } & Fluticasone/salmeterol & 1.00 \\
\hline & Beclometasone dipropionate/formoterol & $0.19[0.16-0.23]^{*}$ \\
\hline \multicolumn{2}{|c|}{ Specialists $(N=289)$} & OR $[95 \% \mathrm{CI}]$ \\
\hline \multirow[t]{2}{*}{ Gender } & Female & 1.00 \\
\hline & Male & $1.19[0.69-2.06]$ \\
\hline \multirow[t]{3}{*}{ Age } & $<18$ years & 1.00 \\
\hline & $18-65$ years & $4.64[1.64-13.13]^{*}$ \\
\hline & $>65$ years & $11.01[2.38-50.88] *$ \\
\hline \multirow[t]{3}{*}{ Comorbidities } & No comorbidities & 1.00 \\
\hline & 1 comorbidity & $0.95[0.48-1.89]$ \\
\hline & At least two comorbidities & $0.65[0.04-9.64]$ \\
\hline PEF & & $1.01[0.99-1.03]$ \\
\hline \multirow[t]{2}{*}{ Smoking } & No & 1.00 \\
\hline & Yes & $1.40[0.75-2.58]$ \\
\hline \multirow[t]{2}{*}{ Medication } & Fluticasone/salmeterol & 1.00 \\
\hline & Beclometasone dipropionate/formoterol & $0.17[0.08-0.37]^{*}$ \\
\hline
\end{tabular}

*, p-value $<0.05$.

BMI: body mass index; DPI: dry powder inhaler; GP: general practitioner; PEF: peak expiratory flow; pMDI: pressurised, metered-dose inhaler; OR: Odds Ratio; CI: confidence interval.

about $41 \%$ of their patients. Among possible causes for poor asthma control, low adherence to therapy was the option to which, on average, specialists assigned the greatest weight (42\%), followed by difficulties in using inhaler devices $(24 \%)$, and severity of the disease $(11 \%)$. It is worth noting that, on average, $53 \%$ and $39 \%$ of patients using pMDIs were reported to experience difficulties in hand-breath coordination "often" or "sometimes", respectively. On the other hand, 20\% and $61 \%$ of the patients using DPIs displayed difficulties in inhaling strongly and deeply "often" or "sometimes", respectively. Specialists reported that, because of the difficulties in using their inhaler devices, the proportion of patients not in their opinion receiving an adequate dose of the drug was, on average, $35 \%$ for those using a pMDI and $21 \%$ for those using a DPI (data not shown). Fig. 2 shows the level of agreement (the higher the score assigned, the higher the level of agreement) among specialists with regard to their opinion about currently available pMDIs and DPIs. Most of the specialists agreed that patients' difficulties with currently available devices could be potential obstacles to achieve full adherence to treatment. Furthermore, the opportunity to prescribe new inhaler devices that may overcome patients' difficulties with current devices seemed to be a popular conception, with $63 \%$ of specialists showing a good or very good level (score $\geq 7$ ) of agreement with this statement (Fig. 2).

When asked to rank the most important characteristic that should be taken into account when choosing a particular device for an asthmatic patient, most (63\%) of the specialists chose "easy use of the device" as being the most important characteristic (data not shown). Fig. 3 shows the mean scores that specialists assigned to various aspects to be considered when choosing an inhaler device for a patient. The drivers of prescriptions to which specialists assigned the highest scores were mainly related to those likely to improve patients' adherence when using an inhaler device (Fig. 3). Furthermore, about $75 \%$ of the specialists agreed that the novel breath-triggered K-haler device might facilitate proper drug intake compared to pMDIs and DPIs; therefore, most of the specialists would likely (43\%) or certainly (45\%) be inclined to prescribe this novel inhaler device (data not shown).

\section{Discussion}

The present study investigated current "real-world" drivers of inhaler choice in asthma patients from the perspective of both primary care and respiratory specialist physicians. The results show that: $i$ ) no meaningful differences exist in the characteristics of patients prescribed pMDIs compared to those prescribed DPIs; $i i)$ the strongest driver of inhaler choice is ICS/LABA medication. We also observed that over a period of 1-year, few patients were prescribed spirometry or specialist examinations, whereas approximately $20 \%$ of patients experienced deterioration in asthma and/or exacerbations. Furthermore, most of the specialists were aware of the difficulties encountered by patients when using pMDIs and DPIs, which were regarded as obstacles to achieving full asthma control. Finally, specialists agreed upon the need for novel inhaler technologies that may overcome patients' difficulties in using 
Table 3

Information collected during 12-month follow-up on GPs' patients stratified by type of device prescribed.

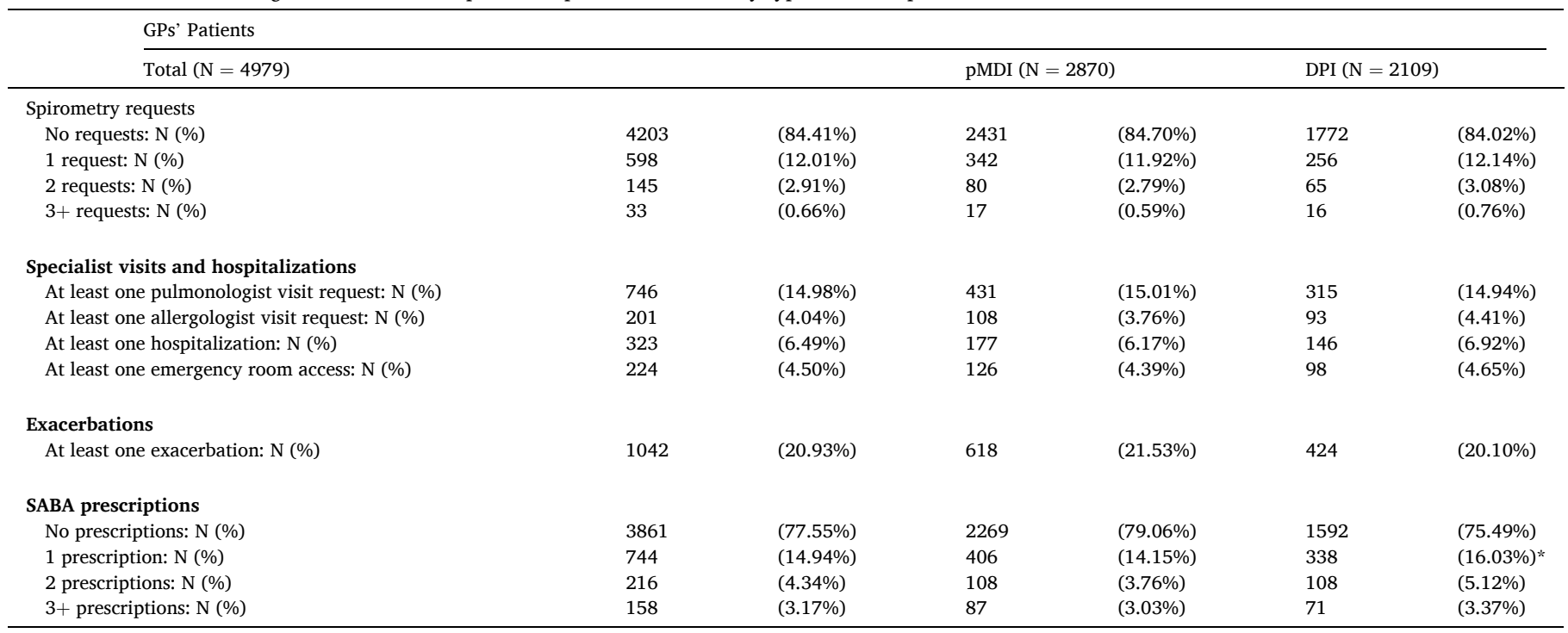

*Statistically significant difference between pMDI and DPI groups: p-value $<0.05$.

DPI: dry powder inhaler; GP: general practitioner; pMDI: pressurised metered-dose inhaler; SABA: short-acting $\beta 2$-agonist.

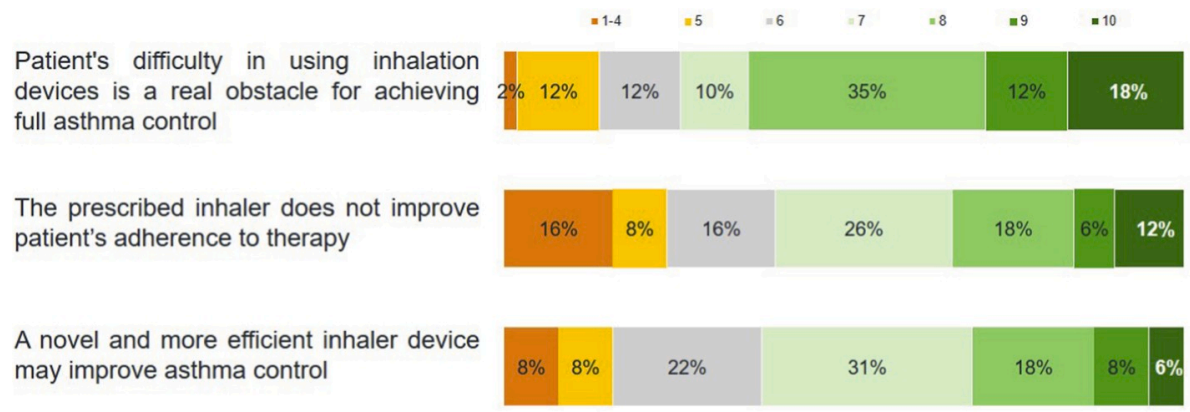

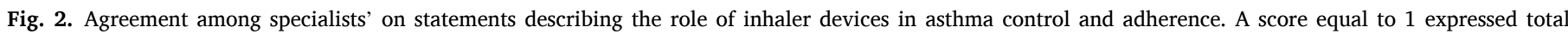
disagreement, whereas a score equal to 10 denotes total agreement.

\begin{tabular}{|l|l|l|}
\hline To improve patients' adherence to treatment & $9,4(1,0)$ \\
\hline To be easy to use & $9,1(1,5)$ & $9,1(1,1)$ \\
\hline To facilitate proper drug administration & $8,9(1,2)$ \\
\hline To ensure good lung deposition of the drug & $8,4(1,8)$ \\
\hline To increase speed of onset of drug effect & $8,2(1,7)$ \\
\hline Being suitable for older patients & $7,8(1,9)$ \\
\hline Being the patient's preferred device & $7,2(2,1)$ \\
\hline Being suitable for younger patients & $7,2(2,0)$ \\
\hline To require short teaching time & 7 \\
\hline
\end{tabular}

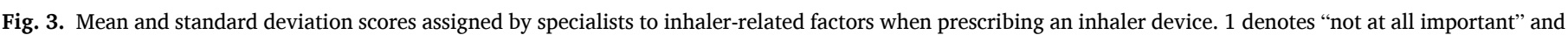
10 denotes "extremely important". 
currently available inhaler devices.

To the best of our knowledge, this is the first study aimed at investigating the behaviour of Italian GPs and respiratory specialists when choosing an inhaler device for asthma patients treated with ICS/LABA medication in a real-life setting. This study adds evidence to the current literature $[17,18]$ and clinical respiratory practice [16] that, in the majority of patients, drug choice is usually the dominant step when prescribing inhaled therapy and, together with drug availability and reimbursement criteria, dictates the inhaler device options. Of note, we found that both GPs and respiratory specialists prescribed ICS/LABA medications via DPIs preferentially to older, smoker and obese patients, and that the fluticasone/salmeterol combination was more likely prescribed via a DPI. Taken together, these findings may suggest a preferential use of the fluticasone/salmeterol combination in patients with potentially more severe disease.

Notwithstanding this current "standard practice", the importance of correct inhaler device selection as a dominant feature of the treatment decision is becoming increasingly recognised [6,9,16,30,31]. Although guidelines have lacked clarity on specific guidance regarding appropriate inhaler selection [32, 33], it is encouraging to see that the latest report on the Global Initiative for Asthma clearly states the importance of inhaler device selection in daily clinical practice (1). Interestingly, several studies have demonstrated a relationship between characteristics of individual inhaler devices and the patient's degree of adherence [8], and that an increased frequency of critical errors in inhalation is associated with worse asthma control, resulting in more disease exacerbations and healthcare resource consumption [11,34]. On the other hand, improvement of inhaler technique by effective training has been associated with better control of symptoms and improved quality of life $[35,36]$. Collectively, these findings indicate that it is indeed reasonable to hypothesise that the selection of an inhaler device tailored to the characteristics of the individual patient results in improved adherence and better clinical outcomes. The importance of factors such as aging, education status, cognitive status, manual dexterity and strength have been highlighted in previous studies [10] and a practical approach to the selection of the most appropriate inhaler device for each patient has been proposed [30,31]. Despite this, the prescribing habit of GPs and respiratory specialists revealed in the present study indicates that they still prioritise the drug over the device when initiating treatment of asthma patients. The reason(s) for this behaviour are not immediately clear, but may reflect ingrained habits rather than a conscious, evidence-based approach.

Although international guidelines do recommend the measuring lung function periodically when managing asthma [1], in the present study few patients managed by general physicians were recalled for spirometry during the 1-year period after initiation of ICS/LABA treatment, independently of the inhaler device prescribed. Similarly, the proportion of patients referred to a respiratory specialist was limited, particularly considering that more than $20 \%$ of the patients experienced at least one exacerbation during this same 1-year period. This finding is particularly meaningful considering that it is comparable to exacerbation rates found in the CRITIKAL study [11], which was specifically aimed at collecting extensive information on asthma patients' demographic characteristics, symptoms, lung function, inhaler technique, and occurrence of exacerbations. Together, these findings suggest sub-optimal management of the disease by family doctors, particularly when it is considered that the patients in this study had been newly commenced on treatment for their disease. In another, community based study, it was shown that only half of the patients with a clinical diagnosis of asthma or COPD had ever performed a spirometry test [37]. Furthermore, a survey involving both GPs and asthma specialists showed that familiarity with, and adherence to asthma guidelines was higher for specialists than for primary care physicians, but was low in both groups for several key recommendations [38].

The majority of respiratory specialists interviewed in the ad-hoc survey agreed upon the tenet that incorrect use of inhaler devices may contribute to poor asthma control, with faults being attributable to pMDIs, as well as to DPIs. Poor confidence in the currently available inhalers by the interviewed specialists may be one of the underlying reasons for the doctors' prescription behaviour observed in the present study. Therefore, it is reasonable to hypothesise that novel inhaler technology, potentially capable of overcoming difficulties currently experienced with DPIs and pMDIs, represents an opportunity to improve asthma control.

Our study has some limitations, the most notable being the inherent selection bias in survey-based retrospective analyses. Firstly, this study was conducted in a single health-care system; therefore, extrapolation of these results to other populations should be with caution. The data do however show trends similar to previous research $[17,18]$. Furthermore, other European countries such as France and Spain share common features with the Italian healthcare system, allowing our results to have important clinical relevance outside Italy. Secondly, the assumption that obtaining a prescription was equivalent to taking the prescribed medication might not be completely accurate [8]. However, rates and volumes of prescriptions by GPs, such as those obtained from the Italian IQVIA LPD, have already been shown to be consistent with those measured by other data sources providing information on dispensed medications [21]. Thirdly, since IQVIA LPD is a secondary data repository developed for purposes other than scientific research, information about hospitalisations is not mandatory and may have been underestimated.

The main strength of the present study is the opportunity to take advantage of very heterogeneous data sources. Using both IQVIA LPD and PA allowed us to gain insight from two different perspectives, that of family doctors and that of respiratory specialists, covering most potential prescribers of asthma treatments. The combined evaluation of evidence obtained from these diverse but complementary real-world data allowed a more comprehensive overview. In addition, deeper insight offered by the ad-hoc survey served as confirmation of the need for new technologies such as that represented by the breath-triggered K-haler device. Our findings unveil GP and specialist perspectives about the role of the inhaler device in asthma treatment. This information could also be used to guide the development of next generation inhaler devices for respiratory disease.

\section{Conclusion}

It has been advocated that the choice of an inhalation device for drug administration in patients with asthma is as critical as the choice of the medication itself [15]. Consequently, the prescriber should be able to select the device based on the patient's characteristics and needs [14]. However, results from the present study suggest a prescription behaviour that seems to be affected by habits, with doctors choosing the type of device in relation to the medicine prescribed, rather than based on patient characteristics.

\section{Declaration of funding}

This study was funded by Mundipharma Pharmaceuticals.

\section{Declaration of competing interest}

To be completed depending on other authors' conflict of interests. V.P. is an employee of IQVIA.

\section{CRediT authorship contribution statement}

F. Lavorini: Conceptualization, Methodology, Writing - original draft. A. Bianco: Conceptualization, Methodology, Writing - original draft. F. Blasi: Conceptualization, Methodology, Writing - original draft. F. Braido: Conceptualization, Methodology, Writing - original draft. A. G. Corsico: Conceptualization, Methodology, Writing - original draft. F. 
Di Marco: Conceptualization, Methodology, Writing - original draft. A. Gentile: Conceptualization, Methodology, Writing - original draft. P.L. Paggiaro: Conceptualization, Methodology, Writing - original draft. V. Pegoraro: Conceptualization, Methodology, Writing - original draft. G. Pelaia: Conceptualization, Methodology, Writing - original draft. P. Rogliani: Conceptualization, Methodology, Writing - original draft. P. Santus: Conceptualization, Methodology, Writing - original draft. N. Scichilone: Conceptualization, Methodology, Writing - original draft. A. Soldi: Conceptualization, Methodology, Writing - original draft. G. W. Canonica: Conceptualization, Methodology, Writing - original draft.

\section{Appendix A. Supplementary data}

Supplementary data to this article can be found online at https://doi. org/10.1016/j.rmed.2020.105937.

\section{References}

[1] Global Initiative for Asthma, Global strategy for asthma management and prevention, Available from: www.ginasthma.org, 2019.

[2] K.R. Murphy, B.G. Bender, Treatment of moderate to severe asthma: patient perspectives on combination inhaler therapy and implications for adherence, J. Asthma Allergy 2 (2009) 63-72.

[3] H.J. van der Woude, M. Boorsma, P.B. Bergqvist, T.H. Winter, R. Aalbers, Budesonide/formoterol in a single inhaler rapidly relieves methacholine-induced moderate-to-severe bronchoconstriction, Pulm. Pharmacol. Therapeut. 17 (2004) 89-95.

[4] F. Braido, et al., Asthma management in a specialist setting: results of an Italian Respiratory Society survey, Pulm. Pharmacol. Therapeut. 44 (2017) 83-87.

[5] F. Braido, et al., "Trying, but failing" - the role of inhaler technique and mode of delivery in respiratory medication adherence, J. Allergy Clin. Immunol. Pract. 4 (2016) 823-832.

[6] F. Lavorini, G.A. Fontana, O.S. Usmani, New inhaler devices - the good, the bad and the ugly, Respiration 88 (2014) 3-15.

[7] D. Price, et al., Inhaler competence in asthma: common errors, barriers to use and recommended solutions, Respir. Med. 107 (2013) 37-46.

[8] J. Darba, et al., Identification of factors involved in medication compliance: incorrect inhaler technique of asthma treatment leads to poor compliance, Patient Prefer. Adherence 135 (2016), https://doi.org/10.2147/PPA.S95303.

[9] F. Lavorini, Inhaled drug delivery in the hands of the patient, J. Aerosol Med. Pulm. Drug Deliv. 27 (2014) 414-418.

[10] P. Rogliani, L. Calzetta, A. Coppola, F. Cavalli, J. Ora, E. Puxeddu, M.G. Matera, M. Cazzola, Optimizing drug delivery in COPD: the role of inhaler devices, Respir. Med. 124 (2017) 6-14.

[11] F. Lavorini, C. Mannini, E. Chellini, G.A. Fontana, Optimising inhaled pharmacotherapy for elderly patients with chronic obstructive pulmonary disease: the importance of delivery devices, Drugs Aging 33 (2016) 461-473.

[12] D.B. Price, M. Román-Rodríguez, R.B. McQueen, S. Bosnic-Anticevich, V. Carter, K. Gruffydd-Jones, J. Haughney, S. Henrichsen, C. Hutton, A. Infantino, F. Lavorini, L.M. Law, K. Lisspers, A. Papi, D. Ryan, B. Ställberg, T. van der Molen, Chrystyn inhaler errors in the CRITIKAL study: type, frequency, and association with asthma outcomes, J. Allergy Clin. Immunol. Pract. 5 (2017) 1071-1081.

[13] S.P. Newman, Dry powder inhalers for optimal drug delivery, Expet Opin. Biol. Ther. 4 (2004) 23-33.

[14] F. Lavorini, M. Pistolesi, O.S. Usmani, Recent advances in capsule-based dry powder inhaler technology, Multidiscip. Respir. Med. 12 (2017).

[15] B. Ding, M. Small, G. Scheffel, U. Holmgren, Maintenance inhaler preference, attribute importance, and satisfaction in prescribing physicians and patients with asthma, COPD, or asthma-COPD overlap syndrome consulting for routine care, Int. J. Chronic Obstr. Pulm. Dis. 13 (2018) 927-936.

[16] F. Lavorini, O.S. Usmani, Correct inhalation technique is critical in achieving good asthma control, Prim. Care Respir. J. 22 (2013) 385-386.

[17] P.N.R. Dekhuijzen, F. Lavorini, O.S. Usmani, Patients' perspectives and preferences in the choice of inhalers: the case for Respimat ${ }^{\circledR}$ or HandiHaler $($ Patient Prefer, Adherence 10 (2016) 1561-1572.
[18] M. Miravitlles, J.J. Soler-Cataluña, B. Alcázar, J.L. Viejo, F. García-Río, Factors affecting the selection of an inhaler device for COPD and the ideal device for different patient profiles. Results of EPOCA Delphi consensus, Pulm. Pharmacol. Therapeut. 48 (2018) 97-103.

[19] N.A. Hanania, S. Braman, S.G. Adams, et al., The role of inhalation delivery devices in COPD: perspectives of patients and health care providers, Chronic. Obstr. Pulm. Dis. 5 (2018) 111-123.

[20] B. Ding, M. Small, G. Scheffel, U. Holmgren, Maintenance inhaler preference, attribute importance, and satisfaction in prescribing physicians and patients with asthma, COPD, or asthma-COPD overlap syndrome consulting for routine care, Int. J. Chronic Obstr. Pulm. Dis. 13 (2018) 927-936.

[21] P. Katz, V. Pegoraro, H. Liedgens, Characteristics, resource utilization and safety profile of patients prescribed with neuropathic pain treatments: a real-world evidence study on general practices in Europe - the role of the lidocaine 5\% medicated plaster, Curr. Med. Res. Opin. 33 (2017) 1481-1489.

[22] M. Levi, et al., Epidemiology of iron deficiency anaemia in four European countries: a population-based study in primary care, Eur. J. Haematol. 97 (2016) 583-593.

[23] M. Ravera, et al., High performance of a risk calculator that includes renal function in predicting mortality of hypertensive patients in clinical application, J. Hypertens. 32 (2014) 1245-1254.

[24] F. Colivicchi, et al., Clinical implications of switching lipid lowering treatment from rosuvastatin to other agents in primary care, Adv. Ther. 33 (2016) 2049-2058.

[25] G. Maina, C. Ripellino, The risk of metabolic disorders in patients treated with asenapine or olanzapine: a study conducted on real-world data in Italy and Spain, Expet Opin. Drug Saf. 13 (2014) 1149-1154.

[26] E. Agostoni, et al., Real-world insights on the management of migraine patients: an Italian nationwide study, Curr. Med. Res. Opin. (2019) 1-10, https://doi.org/ 10.1080/03007995.2019.1602032.

[27] F. Di Marco, et al., Characteristics of newly diagnosed COPD patients treated with triple inhaled therapy by general practitioners: a real world Italian study, Npj Prim. Care Respir. Med. 27 (2017).

[28] E. Visentin, D. Nieri, B. Vagaggini, E. Peruzzi, P.L. Paggiaro, An observation of prescription behaviors and adherence to guidelines in patients with COPD: real world data from October 2012 to September 2014, Curr. Med. Res. Opin. 32 (2016) 1493-1502.

[29] D. Kappeler, K. Sommerer, C. Kietzig, B. Huber, J. Woodward, M. Lomax, P. Dalvi, Pulmonary deposition of fluticasone propionate/formoterol in healthy volunteers, asthmatics and COPD patients with a novel breath-triggered inhaler, Respir. Med. 138 (2018) 107-114.

[30] D. Bell, L. Mansfield, M. Lomax, A randomized, crossover trial evaluating patient handling, preference, and ease of use of the fluticasone propionate/formoterol breath-triggered inhaler, J. Aerosol Med. Pulm. Drug Deliv. 30 (2017) 425-434.

[31] P.N. Dekhuijzen, W. Vincken, J.C. Virchow, N. Roche, A. Agusti, F. Lavorini, W. M. van Aalderen, D. Price, Prescription of inhalers in asthma and COPD: towards a rational, rapid and effective approach, Respir. Med. 107 (2013) 1817-1821, 2013.

[32] R. Dekhuijzen, F. Lavorini, O.S. Usmani, J.F.M. van Boven, Addressing the impact and unmet needs of nonadherence in asthma and chronic obstructive pulmonary disease: where do we go from here? J Allergy Clin Immunol Pract 6 (2018) 785-793.

[33] P.N. Dekhuijzen, L. Bjermer, F. Lavorini, V. Ninane, M. Molimard, J. Haughney, Guidance on handheld inhalers in asthma and COPD guidelines, Respir. Med. 108 (2014) 694-700.

[34] C.J. Corrigan, Asthma therapy: there are guidelines, and then there is real life... Prim. Care Respir. J. 20 (2011) 13-14.

[35] A.S. Melani, M. Bonavia, V. Cilenti, C. Cinti, M. Lodi, P. Martucci, M. Serra, N. Scichilone, P. Sestini, M. Aliani, M. Neri, Inhaler mishandling remains common in real life and is associated with reduced disease control, Respir. Med. 105 (2011) 930-938.

[36] A. Dudvarski Ilic, V. Zugic, B. Zvezdin, I. Kopitovic, I. Cekerevac, V. Cupurdija, N. Perhoc, V. Veljkovic, A. Barac, Inhaler technique on asthma and COPD control: a multicenter experience, Int. J. Chronic Obstr. Pulm. Dis. 11 (2016) 2509-2517.

[37] E. Heffler, et al., Misdiagnosis of asthma and COPD and underuse of spirometry in primary care unselected patients, Respir. Med. 142 (2018) 48-52.

[38] M.M. Cloutier, et al., Clinician agreement, self-efficacy, and adherence with the guidelines for the diagnosis and management of asthma, J. Allergy Clin. Immunol. Pract. 6 (2018), 886-894.e4. 